\title{
Small Intestinal Submucosa Matrix as a Novel Therapy for Wounds in Dystrophic Epidermolysis Bullosa
}

\author{
Isaacs, Michael MD; Veerkamp, Patrick MD; Somani, Ally-Khan MD, PhD \\ Department of Dermatology, Indiana University School of Medicine, Indianapolis, Indiana
}

The authors have indicated no significant interest with commercial supporters.

Recessive dystrophic epidermolysis bullosa (RDEB) is a rare, hereditary disease of the skin, which manifests as skin fragility, widespread bulla, and erosions that heal with significant scarring and contracture. This condition is the result of a COL7A1 gene mutation that encodes Type VII collagen. Patients with RDEB are at increased risk for the development of cutaneous squamous cell carcinoma (SCC).1 The first-line treatment for SCC in RDEB is surgery, but the postsurgical defect poses a challenge for repair given the recurrent blistering and erosions. The skin defect has traditionally been left to heal by secondary intention or repaired with split or full-thickness skin grafts, 2 which have the disadvantages of limited normal skin for grafting and the potential for prolonged healing of the harvest site. We introduce the porcine small intestinal submucosa (SIS) matrix as a novel reconstructive option for the repair of surgical defects or nonsurgical wounds in patients with RDEB.

Small intestinal submucosa matrix has been successfully used 17 times on the extremities of 2 patients with RDEB in our clinic, each time after Mohs micrographic surgery (MMS) for the treatment of an SCC. Patient 1, a 46-year-old man with Hallopeau-Siemens type RDEB, was treated 12 times. Patient 2, a 43-year-old woman, was treated 5 times. After an average of 2.0 stages of

This is the author's manuscript of the work published in final form as:

Isaacs, M., Veerkamp, P., \& Somani, A.-K. (2019). Small Intestinal Submucosa Matrix as a Novel Therapy for Wounds in Dystrophic Epidermolysis Bullosa. Dermatologic Surgery: Official Publication for American Society for Dermatologic Surgery [et Al.], 45(6), 863-864.

https://doi.org/10.1097/DSS.0000000000001731 
MMS, postsurgical defects ranged in size from 5 to $62 \mathrm{~cm}^{2}$ (mean of $16.8+/-15.7 \mathrm{~cm}^{2}$ ). Each defect was allowed to heal by secondary intention facilitated by the placement of porcine SIS matrix in the wound bed, adhered to surrounding skin using steristrips (Figure 1), and covered in a thin layer of petrolatum ointment and a pressure dressing. A bed of granulation tissue covered each defect at 4 weeks, and all wounds healed in an average of 6.0 weeks (range 5.5-7.0 weeks). Furthermore, none of the defects treated with SIS resulted in chronic wounds, which is commonly observed in RDEB wounds in general.3 Both patients reported good functional and cosmetic outcomes (Figure 1).

The wound-healing environment in patients with RDEB is unfavorable. Therefore, a product that mimics the native extracellular matrix (ECM) and provides scaffolding for the ingrowth of cells could be highly beneficial. The porcine SIS matrix (OASIS and OASIS Ultra; Cook Biotech Inc., West Lafayette, IN) we used is especially appealing for patients with RDEB because it interacts with the native epidermis and leads to accelerated regeneration of basement membrane components including Type VII collagen,4 the same protein that is dysfunctional or absent in RDEB. We postulate this mechanism along with providing an intact ECM in the wound is likely responsible for the increased integrity (stabilization of anchoring fibrils) and accelerated healing of the skin in patients with mutated or absent Type VII collagen treated with porcine SIS matrix. In addition, the SIS matrix contains other important components found in the human dermis such as elastin, glycosaminoglycans, glycoproteins, proteoglycans, fibroblast growth factor, and transforming growth factor [beta]. Furthermore, SIS matrix is easy to apply as described by Cazzell and colleagues. 5

The porcine SIS matrix is an effective alternative reconstruction option for patients with RDEB. It is ideal for these patients because it enhances regeneration of Type VII collagen through interaction with the epidermis and avoids further manipulation of their fragile skin. Porcine SIS matrix has utility in healing wounds in patients with RDEB and should be considered as a viable option for treatment of surgical and nonsurgical wounds alike in patients with RDEB. 


\section{References}

1. Fine JD, Johnson LB, Weiner M, Li KP, et al. Epidermolysis bullosa and the risk of life-threatening cancers: the National EB Registry experience, 1986-2006. J Am Acad Dermatol 2009;60:203-11.

2. Yamada M, Hatta N, Sogo K, Komura K, et al. Management of squamous cell carcinoma in a patient with recessive-type epidermolysis bullosa dystrophica. Dermatol Surg 2004;30:1424-9.

3. Cianfarani F, Zambruno G, Castiglia D, Odorisio T. Pathomechanisms of altered wound healing in recessive dystrophic epidermolysis bullosa. Am J Pathol 2017;187:1445-53.

4. Lindberg K, Badylak SF. Porcine small intestinal submucosa (SIS): a bioscaffold supporting in vitro primary human epidermal cell differentiation and synthesis of basement membrane proteins. Burns 2001;27:254-66.

5. Cazzell SM, Lange DL, Dickerson JE, Slade HB. The management of diabetic foot ulcers with porcine small intestine submucosa tri-layer matrix: a randomized controlled trial. Adv Wound Care 2015;4:711-8. 
Figure

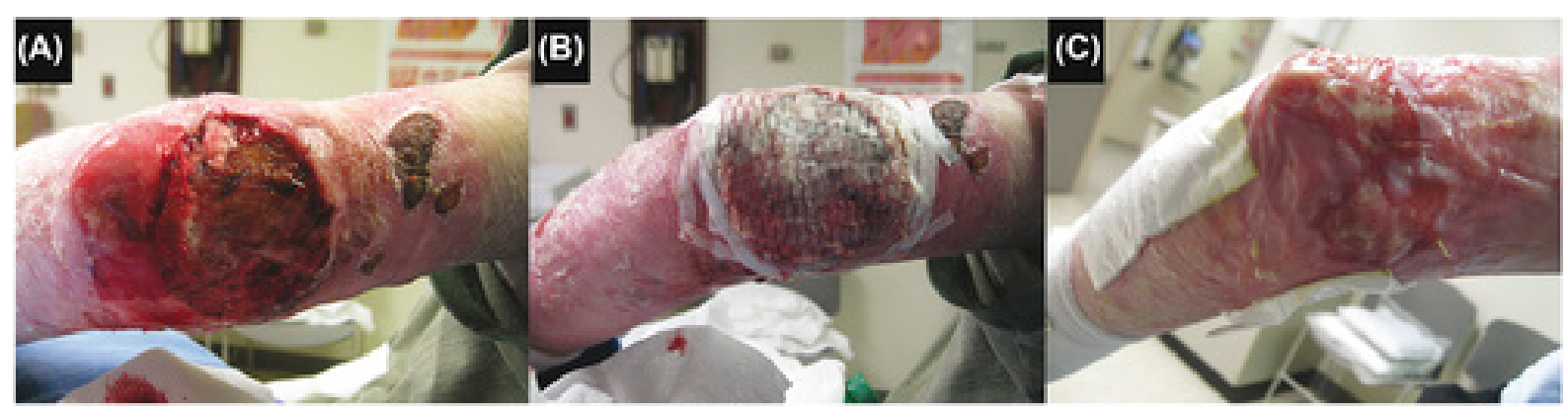

Figure 1. RDEB patient underwent Mohs micrographic surgery for recurrent SCC on the left elbow. (A) The tumor was cleared with 2 stages resulting in a $27-\mathrm{cm}^{2}(6.0 \times 4.5-\mathrm{cm})$ defect down to deep subcutaneous tissue. (B) Placement of porcine SIS matrix (Oasis Ultra) in the wound bed. (C) Six-week follow-up demonstrates accelerated healing and wound reepithelization. RDEB, recessive dystrophic epidermolysis bullosa; SCC, squamous cell carcinoma; SIS, small intestinal submucosa. 